\title{
Numerical Study on Improving the Efficiency of the Earth Pipe Cooling System
}

\author{
Parnab Saha ${ }^{1}$, Musanna Galib ${ }^{1}$, Md. Mahdi-Ul-Ishtiaque ${ }^{1}$, Shamimur Rahman Akanda ${ }^{1}$ and \\ Mohammed Abdul Hannan ${ }^{2 *}$ \\ ${ }^{1}$ Bangladeh University of Engineering \& Technology, Dhaka, Bangladesh \\ ${ }^{2}$ Faculty of Science, Agriculture \& Engineering, Newcastle University, UK (Singapore Unit), \\ Singapore.
}

\begin{abstract}
Earth pipe cooling (EPC) is one of the passive cooling systems which has proven to be a very energy efficient sustainable cooling technique for the sub-tropical areas. This cooling system uses the soil as a medium of heat transfer. Efficiency of this system largely depends on the velocity of the cooling air, shape of the cooling space, pattern of the walls in cooling space, inlet size and position of the incoming air. It is wellknown that increasing the velocity of the cooling air will improve the efficiency of the cooling system. Therefore, this study focused on the optimization of the shape and pattern of the inlet which might improve the efficiency of the cooling system. ANSYS Fluent is used to perform this simulation-based study. It is found that inclusion of aerofoil shaped turbulator in the inlet cross sectional area provide better circulation of the air inside the cooling space, thus, enhance the effectiveness of the system.
\end{abstract}

\section{Introduction}

Consumption of energy in a feasible and efficient way is one of the main concerns in developing sustainable lifestyles. With the rapid growth of population, energy demand is increasing drastically. Analyzing the trend of increase in population, it is estimated that world population will be around 8.7 billion by 2035 which will add around extra $18.4 \%$ to the total current population [1]. Such a prediction poses a clear threat to the sustainability of the world. Therefore, it is of cardinal concern to find out alternative sustainable energy sources to fulfill the energy gap in various sectors. For example, the efficient, environmental friendly and cost-effective use of air conditioning and refrigeration system is getting complicated and difficult to attain day by day. Passive air cooling system in this case can be an effective alternative energy source to the traditional air-conditioning system and can save substantial amount of energy.

One on the passive air cooling methods is the Earth pipe cooling (EPC) system. In this method, atmospheric air is entered in a pipe passing through the Earth-soil. This pipe acts as a heat exchanger between the air in a closed space on the Earth surface and the Earthsoil. The hot air enters the pipe beneath the Earth and release the heat to the cooler soil. The

\footnotetext{
* Corresponding author: abdul.hannan@newcastle.ac.uk
} 
comparatively colder air returns to the closed space above the Earth surface to complete the cycle. The sketch of a simplified EPC system is shown in Fig. 1. If air is not cooled enough, the temperature can be further reduced by the use of interconnected air-conditioning system.

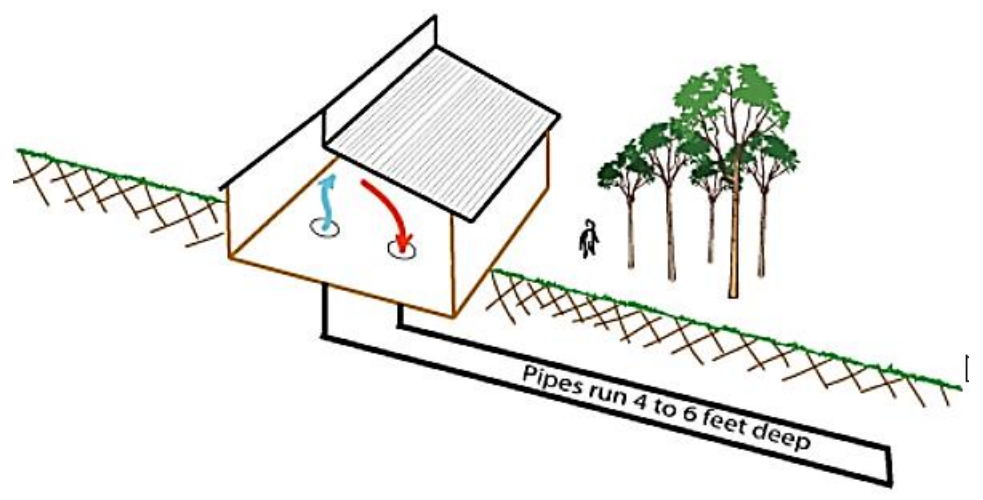

Fig. 1. Sketch of a simplified passive earth pipe cooling system (source: http://www.resilience.org)

Passive cooling technologies are one of the major architectural elements for zero energy building. Significant numbers of researches have already been conducted to measure and improve the effectiveness of the various passive cooling systems. These technologies provide clean, comfortable energy at a relatively lower expense and also having lowest environmental impact. Performance of most of the passive cooling technologies for example, phage change materials (PCM), EPC system largely depends on daily temperature and humidity [2]. PCM improve the thermal properties of building material which plays a vital role in reducing the cooling load [3]. Past research have shown that due to use of PCM reduction a reduction of $11-25 \%$ in peak-load can be achieved [4].

EPC technology on the other hand, consists of a series of buried pipes where the ground acts as heat sink. Channeled air from atmosphere exchanges heat with ground via convection [5]. Additionally, use of heat pump can increase the coefficient of performance (COP) by assisting the heat transfer from the air in the buried pipe [5]. A recent case study with horizontal earth pipe cooling system (HEPC) and vertical earth pipe cooling system (VEPC) indicates that for a particular size, the room temperature can be reduced up to $1.05^{\circ}$ $\mathrm{C}$ and $1.82^{\circ} \mathrm{C}$ respectively. The maximum annual savings that can be achieved by these HEPC and VEPC are $7.93 \%$ and $8.82 \%$ of the total cost which clearly indicates the costeffectiveness of this environment-friendly system [6].

Typically, properties such as mass flow rate of air, pipe radius, pipe length, depth of pipe have significant impact on the thermal performance of EPC system. With the increase of depth of buried pipe up to $4 \mathrm{~m}$, outlet air temperature decreases and COP increases gradually. Larger diameter pipes give more heat transfer surface area and decreasing pipe diameter makes channeled air to perform convection with ground more frequently [7]. For measuring the thermal performance, a transient model was developed and studied in Southern China using PHOENICS [8] which achieved a cooling capacity of up to 74.6 $\mathrm{KW} / \mathrm{h}$. Several studied have shown the potential of EPC in hot humid climates. A theoretical model showed that EPC system can reduce typical house cooling energy up to $30 \%$ in summer [9]. An agricultural greenhouse in Thailand implemented HEPC to cool the house during day time [10].

As can be seen, significant efforts have been provided to design and analyze the EPC system. However, not much emphasis is given yet towards the optimization of the system performances. Therefore, the objective of this study is to shed further light on improving the efficiency of the existing EPC system via modification of the target cooling area and the 
inlet of the cooling air. Aerofoil shaped turbulators are used near the inlet to improve the circulation of the incoming cooling air and significant results have been obtained. The next few sections of this paper describe the configuration of the numerical setup adopted in this study and the validation of the model with experimental analysis, followed by the discussion on the design modification of the inlet and associated results obtained.

\section{Numerical configuration and validation}

\subsection{Numerical setup}

A CFD based turbulence model was solved using finite volume method in "ANSYS 16.2 Fluent solver". EPC is basically a heat transfer process. Therefore, the $\mathrm{k}-\varepsilon$ turbulence model which satisfies the physics of turbulent flow is used to simulate the EPC system analyzed in this study. A three-dimensional density-based solver with absolute velocity formation along with planer space was used for the numerical calculation of the model. Governing equations selected for this purpose are taken to be solved sequentially. Planer 3D space and steady time have been assumed primarily. PRESTO scheme is used for pressure terms discretization, momentum, turbulent kinetic energy and turbulent dissipation rate have been obtained via spatial discretization of first order upwind scheme to obtain accurate results of the simulation.

The convergence criteria are set as $1 \mathrm{e}-6$ for all residuals. The second order implicit discretization scheme is used and to ensure the stability of the solution.

\subsection{Numerical model}

In this study a container shape domain of $5.63 \times 2.14 \times 2.26 \mathrm{~m}$ dimension is adopted as the cooling space which has an inlet having diameter of $0.125 \mathrm{~m}$. This system is similar to the EPC experimental system used by Ahmed et al [6]. The numerical model of the present study will be validated with this experimental study in the later section of this paper, followed by the optimization study performed by adding aerofoil shaped turbulators near the inlet of this same numerical model. Fig. 2 shows the model developed in ANSYS Fluent. Here, the location of the inlet is at $(0.68,0.0,1.113)$ with respect to the coordinate origin.

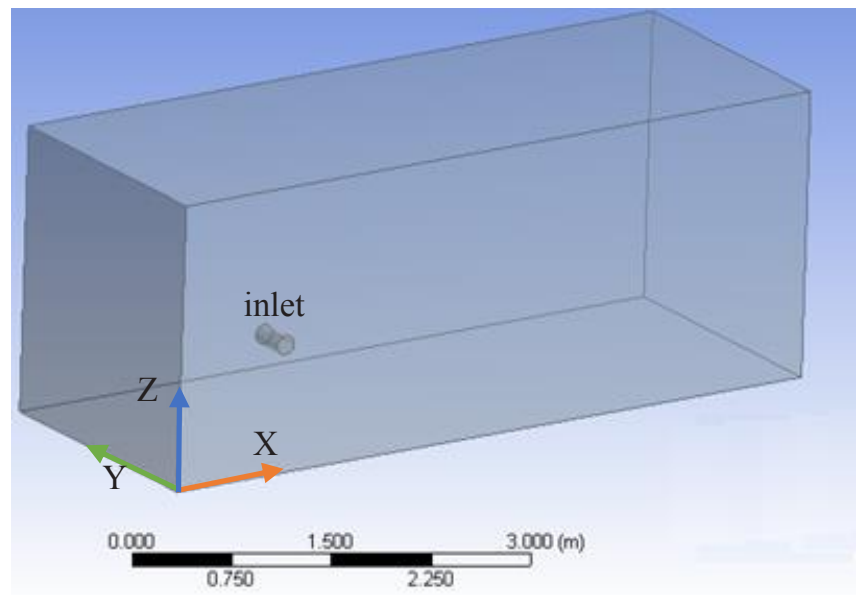

Fig. 2. Numerical model with the coordinate system 


\subsection{Grid sensitivity study}

The initial step of the numerical analysis presented in this paper was to obtain a mesh independent result. In order to do so, the effect of grid variation is studied to obtain the optimum mesh size. A total of 5 cases were carried out for a single value of inlet air velocity $(\mathrm{m} / \mathrm{s})$. The entire simulation domain is then divided into 8 equal segments and the air velocity right at the middle of all these segments are compared for the 5 cases. The results are shown in Table 1 . As can be seen, both the $147 \mathrm{~K}$ and $175 \mathrm{~K}$ node cases exhibits similar air velocity across all the segments. Therefore, $147 \mathrm{~K}$ number of nodes are used in rest of the simulations.

Table 1. Comparison of air velocity at different locations of the cooling space

\begin{tabular}{|c|c|c|c|c|c|c|c|c|}
\hline \multirow[b]{2}{*}{$\begin{array}{l}\text { Number } \\
\text { of Nodes }\end{array}$} & \multicolumn{8}{|c|}{ Coordinates of the mid points of eight segments of the entire domain (m) } \\
\hline & $\begin{array}{l}X=1.075 \\
Y=0.532 \\
Z=0.565\end{array}$ & $\begin{array}{l}X=1.075 \\
Y=0.532 \\
Z=1.695\end{array}$ & $\begin{array}{l}X=4.225 \\
Y=0.532 \\
Z=0.565\end{array}$ & $\begin{array}{l}X=4.225 \\
Y=1.597 \\
Z=1.695\end{array}$ & $\begin{array}{l}X=1.407 \\
Y=1.597 \\
Z=\mathbf{0 . 5 6 5}\end{array}$ & $\begin{array}{l}X=1.407 \\
Y=1.597 \\
Z=1.695\end{array}$ & $\begin{array}{l}X=4.225 \\
Y=1.597 \\
Z=0.565\end{array}$ & $\begin{array}{l}X=4 . .225 \\
Y=1.597 \\
Z=1.695\end{array}$ \\
\hline $50 \mathrm{k}$ & 0.0283 & 0.0315 & 0.0206 & 0.0089 & 0.0434 & 0.0447 & 0.0169 & 0.0145 \\
\hline $75 \mathrm{k}$ & 0.0146 & 0.0372 & 0.0256 & 0.0092 & 0.0248 & 0.4194 & 0.0080 & 0.0210 \\
\hline $103 \mathrm{k}$ & 0.0209 & 0.0216 & 0.0206 & 0.0138 & 0.0313 & 0.0496 & 0.0067 & 0.0256 \\
\hline $143 \mathrm{k}$ & 0.0300 & 0.0302 & 0.0107 & 0.0270 & 0.0396 & 0.0423 & 0.0233 & 0.0096 \\
\hline $175 \mathrm{k}$ & 0.0284 & 0.0288 & 0.0108 & 0.0280 & 0.0345 & 0.0422 & 0.0189 & 0.0087 \\
\hline
\end{tabular}

\subsection{Validation of the numerical model}

As already mention in section 2.2, the numerical model developed for the current simulation study is validated against an experimental analysis conducted by [6]. In one of their experimental study, they used a setup consists of horizontal buried pipes connected to a shipping container (the cooling space) and it was installed at central Queensland University, Australia. In this Horizontal Earth Pipe Cooling (HEPC) system, the container was fitted with two PVC pipe of outside diameter $0.125 \mathrm{~m}$ which works as the manifolds. Intake air comes through one of this manifold and passes through a series of 20 corrugated buried pipes aligned horizontally and moves into the room through another manifold marked as inlet in Fig. 2. Atmospheric air to earth soil heat conduction in this system happens due to temperature differences. Trees are planted to shed the soil to reduce the absorption of solar energy. ReedVane Anemometer and HOBO Pendant Temp Logger were used to find air velocity and temperature of the experimental setup. The associated boundary conditions with this experimental setup is shown in Table 2. These same parameters are used to setup the numerical model for validation. 
Table 2. Boundary conditions for the experimental and simulation model

\begin{tabular}{|c|c|}
\hline Parameters & Value \\
\hline Inlet velocity & $1.1 \mathrm{~m} / \mathrm{s}$ \\
\hline Inlet Temperature & 20.05 degree \\
\hline Room Temperature & 24.90 degree \\
\hline Air Thermal Conductivity & $0.024 \mathrm{~W} /(\mathrm{m} \mathrm{K})$ \\
\hline Air Density & $1.204 \mathrm{~kg} / \mathrm{m}^{2}$ \\
\hline Specific Heat of air & $1006.43 \mathrm{~J} / \mathrm{kg} . \mathrm{k}$ \\
\hline Air viscosity & $1.850387 \mathrm{e}-05 \mathrm{~kg} / \mathrm{m} . \mathrm{s}$ \\
\hline
\end{tabular}

The room temperatures obtained from the simulation is compared with the corresponding experimental results at various heights along the center line of the cooling space and the comparison results are shown in Table 3.

Table 3. Comparison between the experimental and numerical room temperature

\begin{tabular}{|c|c|c|}
\hline Height (m) & Experimental Temp (deg) & Numerical Temp (deg) \\
\hline 0.25 & 25.49 & 24.71 \\
\hline 0.50 & 24.93 & 23.89 \\
\hline 0.75 & 24.67 & 23.78 \\
\hline 1.00 & 24.51 & 23.67 \\
\hline 1.25 & 24.84 & 23.86 \\
\hline 1.50 & 24.58 & 23.98 \\
\hline 1.75 & 24.6 & 23.87 \\
\hline 2.00 & 24.19 & 23.72 \\
\hline
\end{tabular}

As noticed, the simulation results show some deviations from the experimental results which might occurred due to several reasons, for example, uncertainties associated with the experimental measurements, errors associated with the numerical model setup. One of the major differences between the experimental setup and simulation model is that the horizontal buried pipes are not numerically simulated. Only the cooling space, along with the inlet is modelled as the focus of this initial study is to establish the effectiveness of the proposed optimization method rather than numerically simulating the entire experimental HEPC system. However, as shown in Fig. 3 the overall agreement between the simulated results and experimental outcome is quite reasonable and the percentage of differences are between $4.17 \%$ to $1.94 \%$.

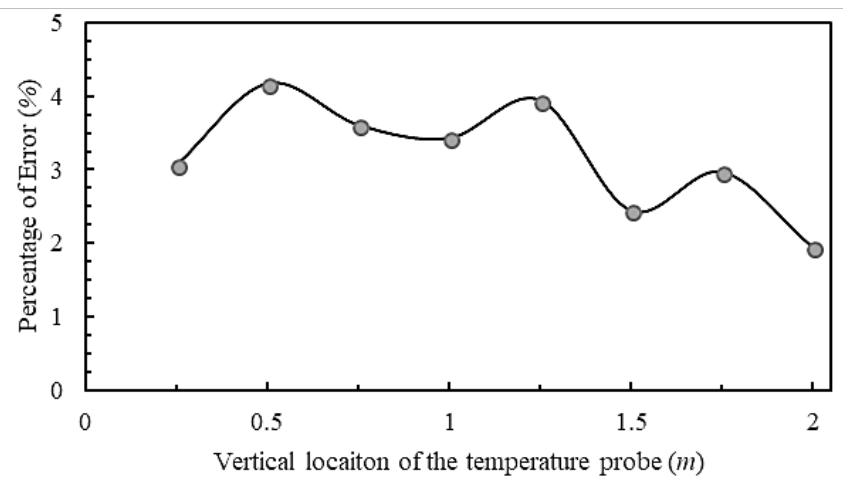

Fig. 3. Percentage of differences between the numerical and experimental results 


\section{System optimization, results and discussion}

In this section, aerofoil shaped turbulator is introduced near the inlet to improve the cooling efficiency of the existing HEPC system. A parametric study is performed by varying the number of turbulators and arrangement of turbulators while other parameters are kept fixed as in Table 2. Fig. 4 below shows the entire cooling space with 16 number of turbulators and also shows the close-up view of the arrangement of the turbulators.
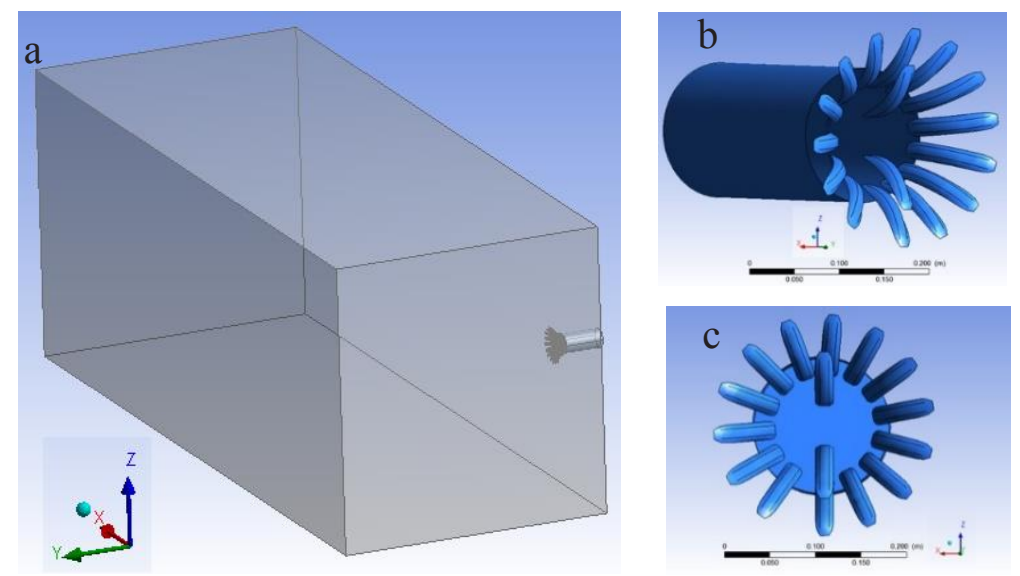

Fig. 4. (a) Simulation domain with 16 turbulator near the inlet; Close up (b) 3 D view; (c) 2 D view of the turbulator arrangement.

The corresponding mesh is shown in Fig. 5. Hex dominant mesh is used in this case with a total number of node as $147 \mathrm{~K}$ and $770 \mathrm{~K}$ elements. It should be noted here that ideally a rather increased number of nodes should be used for a more improved results.

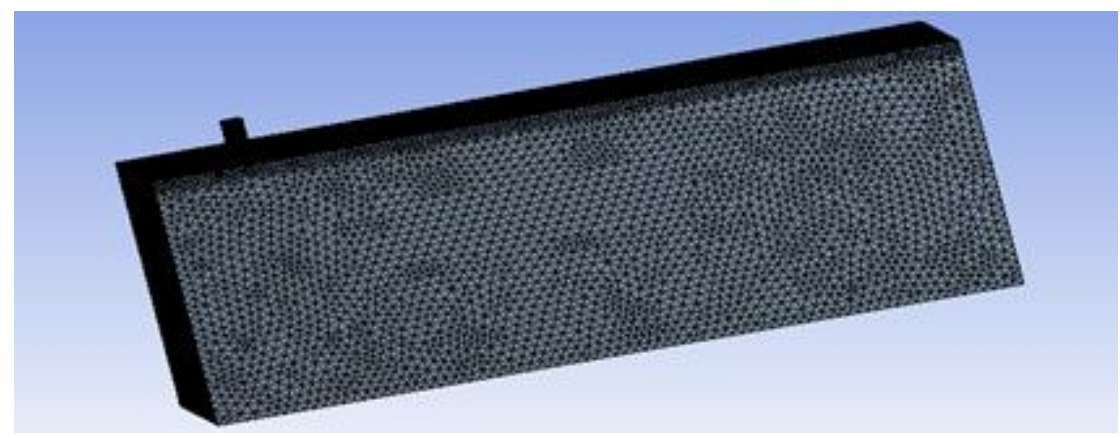

Fig. 5. Meshed domain of the simulation model

The setup and boundary conditions for these simulation studies with turbulator are kept the same as in section 2.4. The results obtained from the simulations using different number of turbulators are tabulated in Table 4 in terms of temperature variations across 5 different points inside the domain. 
Table 4. Variation of temperatures inside the domain with different turbulator arrangements

\begin{tabular}{|c|c|c|c|c|c|c|}
\hline \multirow[b]{2}{*}{$\begin{array}{l}\text { Number of } \\
\text { turbulators }\end{array}$} & \multicolumn{5}{|c|}{ Coordinates of 5 selected points inside the domain (m) } & \multirow[b]{2}{*}{$\begin{array}{c}\text { Avg Temp } \\
\text { inside the } \\
\text { domain (deg) }\end{array}$} \\
\hline & $\begin{array}{c}X=2.81 \\
Y=1.07 \\
Z=0.5\end{array}$ & $\begin{array}{l}X=2.81 \\
Y=1.07 \\
Z=1.00\end{array}$ & $\begin{array}{l}X=2.81 \\
Y=1.07 \\
Z=1.50\end{array}$ & $\begin{array}{l}X=\mathbf{2 . 8 1} \\
Y=\mathbf{1 . 0 7} \\
Z=\mathbf{2 . 0 0}\end{array}$ & $\begin{array}{l}X=2.81 \\
Y=1.07 \\
Z=2.25\end{array}$ & \\
\hline 0 & 23.89 & 23.67 & 23.98 & 23.72 & 23.7 & 23.860 \\
\hline 4 & 23.981 & 23.848 & 23.999 & 23.819 & 23.630 & 23.665 \\
\hline 8 & 24.229 & 24.094 & 24.223 & 24.142 & 23.956 & 23.935 \\
\hline 12 & 24.034 & 23.914 & 23.957 & 23.889 & 23.673 & 23.831 \\
\hline 16 & 23.793 & 23.898 & 23.879 & 23.651 & 23.720 & 23.560 \\
\hline 20 & 23.847 & 23.826 & 23.939 & 23.904 & 23.662 & 23.574 \\
\hline 28 & 24.013 & 23.856 & 24.035 & 23.960 & 23.698 & 23.571 \\
\hline
\end{tabular}

In order to maintain the consistency in the comparison, these 5 points are selected from the 8 points already defined in Table 3 . The temperature profiles summarized in Table 4 are shown in Fig. 6 as well to ensure a better visual representation. As noticed, the temperature profile is varying quite abruptly with the change of turbulator numbers. That means it is rather difficult to conclude on the efficiency of turbulator by comparing the temperatures only on these 5 points inside this large domain.

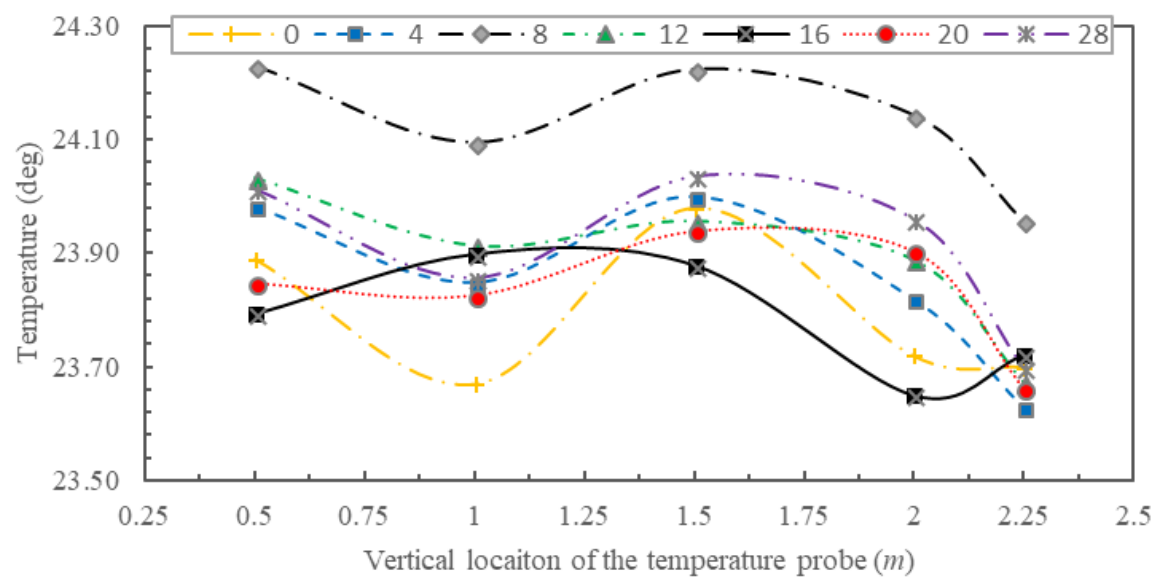

Fig. 6. Temperature profile at different height for various number of turbulators

Comparison of the average room temperatures for different number of turbulators (as shown in Fig 7) appears to provide much meaningful insights. Such comparison clearly shows that inlet with 16 and 20 turbulators generates better cooling effects and increasing the number of turbulators beyond that has no significant impact. It is also observed that addition of 4 turbulators provides significant improvements as well, when compared with the base case (zero turbulator, i.e. the validation case). However, 8 and 12 turbulators cases, especially 8 turbulators, shows unexpected results which might be due to the turbulators arrangement configuration chosen for this simulation case. 


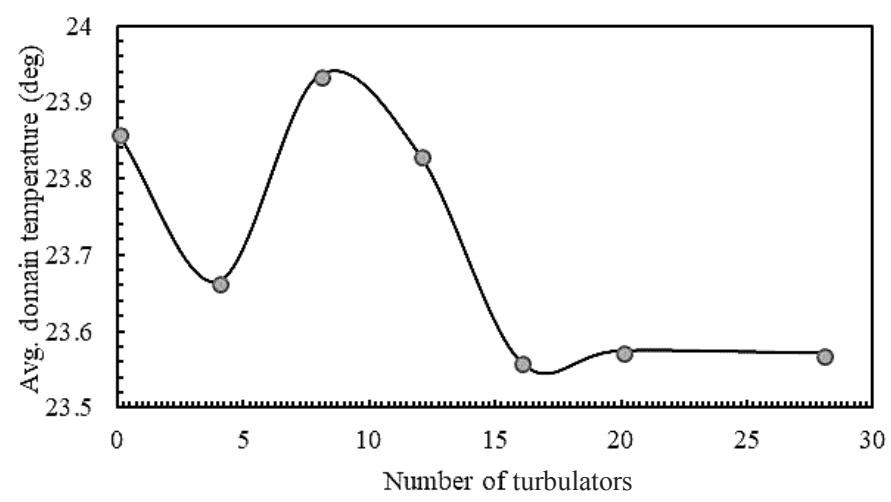

Fig. 7. Variation of average room temperature with the change of turbulator number

Finally, the temperature contour plots for 16 turbulators case and without (zero) turbulator case are compared in Fig. 8 which clearly shows how the presence of turbulators is helping to diverge the flow inside the domain, thus improving the cooling efficiency of the system. These two temperature contours are taken as parallel to $x z$ plane and at a location of $y=1.04 m$.
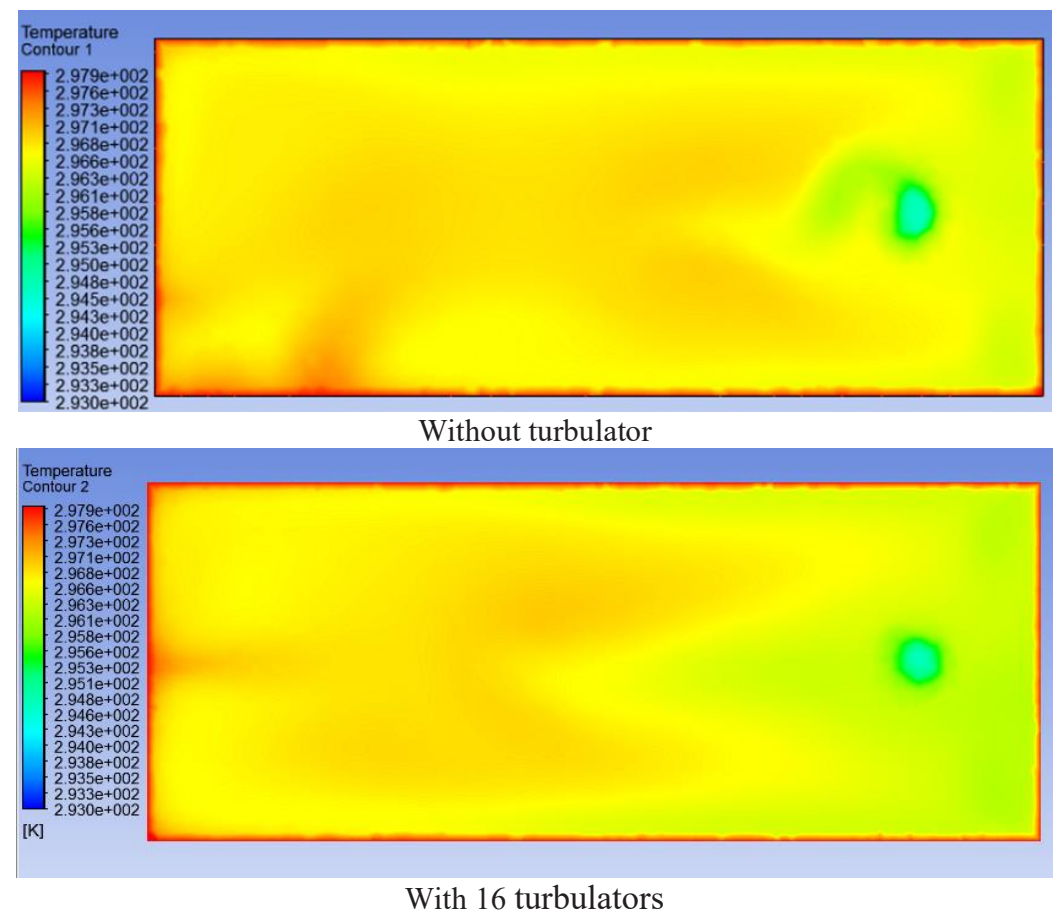

Fig. 8. Temperature contour showing the air circulation at a particular plane

\section{Conclusion}

A method of improving the cooling efficiency of VEPC system by modifying the flow of cooling air near the inlet is presented. The reference experimental analysis shows a 
decrease of 0.32 degree in domain temperature (for a particular setup) due to the effect of VEPC and the corresponding numerical study described in this paper shows an additional decrease of 0.20 degree for that same setup which is achieved by improving the circulation of the inlet air through the application of aerofoil shaped turbulators.

However, the numerical model presented here is still under study and more details parametric study will be conducted to achieve an accurate conclusion on the performances of the proposed method of improving the efficiency. Nevertheless, this study has evidently demonstrated the effectiveness of the idea of adding aerofoil shape near the inlet following a proper procedure to increase the efficiency of the EPC passive cooling system. The improvement on the efficiency achieved in this way will certainly help to save significant amount of energy every year.

\section{References}

1. BP (British Petroleum), BP Energy Outlook 2035 (Published online), United Kingdom (2015)

2. M. Cucumo, S. Cucumo, L. Montoro, A. Vulcano, A one-dimensional transient analytical model for earth-to-air heat exchangers, taking into account condensation phenomena and thermal perturbation from the upper free surface as well as around the buried pipe, int. j. heat mass transfer, 51, 506-516 (2008)

3. German Energy Solutions Initiative, Energy-efficient heating and cooling with phasechange materials (Published online), (2018)

4. M. I. Hasan, H. O..Basher, A. O..Shdhan, Experimental Investigation of Phase Change Materials for Insulation of Residential Buildings, Sustainable Cities and Society, 36, 42-58 (2018)

5. S F Ahmed, M M K Khan, M T O Amanullah, M G Rasul, and N M S Hassan, Performance analysis of vertical earth pipe cooling system for subtropical climate, 13th International Conference on Clean Energy, (2014)

6. S. F. Ahmed, M. M. K. Khan, M. T. O. Amanullah, M. G. Rasul, and N. M. S. Hassan, Performance assessment of earth pipe cooling system for low energy buildings in a subtropical climate, Energy Convers. Manag., 106, 815-825 (2015)

7. M. K. Ghosal and G. N. Tiwari, Modeling and parametric studies for thermal performance of an earth to air heat exchanger integrated with a greenhouse, Energy Convers. Manag., 47, 1779-1798, (2006)

8. H. Wu, S. Wang, and D. Zhu, Modelling and evaluation of cooling capacity of earthair-pipe systems, Energy Convers. Manag., 48, 1462-1471, (2007)

9. F. Al-Ajmi, D. L. Loveday, and V. I. Hanby, The cooling potential of earth-air heat exchangers for domestic buildings in a desert climate, Build. Environ., 41 (3), 235244, (2006)

10. S. Mongkon, S. Thepa, P. Namprakai, and N. Pratinthong, Cooling performance assessment of horizontal earth tube system and effect on planting in tropical greenhouse, Energy Convers. Manag., 78, 225-236, (2014) 